\title{
Microwave assisted synthesis of 2,5-distyryl-1,3,4- oxadiazole derivatives as anti microbial agents
}

\author{
Dipti L. Namera, Jaynt B. Rathod, Rupali H. Maheta, Umed C. Bhoya* \\ Chemical Research Laboratory, Department of Chemistry, Saurashtra University, \\ Rajkot - 360005, Gujrat, India \\ *E-mail address: drucbhoya@gmail.com
}

\begin{abstract}
A new series of 2,5-distyryl-1,3,4-oxadiazoles derivatives have been synthesized from cinnamic hydrazide on reaction with various cinnamic acid derivatives. The structures of synthesized compounds have been elucidated by spectral studies like IR, ${ }^{1} \mathrm{HNMR}$, Mass and also Elemental Analysis. Furthermore, all synthesized compounds were screened for in vitro anti microbial activity against the gram positive (Staphylococcus aureus, Pseudomonas aeruginosa) and gram negative (Escherichia coli) bacterial strain. In which some the compounds show potential inhibition against the test organisms.
\end{abstract}

Keywords: Substituted cinnamic acid; Substituted 1,3,4-oxadiazoles; antimicrobial activity

\section{INTRODUCTION}

Oxadiazole exists in different isomeric form such as $1,2,4 ; 1,2,3 ; 1,2,5$ and 1,3,4. Particularly 1,3,4-oxadiazole ring was thermally stable and have played an important role in medicinal chemistry. They are building blocks for the synthesis of biologically active scaffold. Various remarkable biological activity of 1,3,4-oxadiazoles derivative were reported in literature such as antimicrobial [1-3], antifungal [4,5], anticonvulsant [6], Monoamine oxidase inhibitor [7] and anti-proliferative [8], Hypoglycemic [9] and also they are very good bio isosteres of esters and amides, which can contribute significantly in increasing pharmacological activity by in hydrogen bonding interaction with the receptors [10]. B. Narayana and et al have reported synthesis via modified Fischer Indole method and the antiinflammatory activity [11]. The synthetic adaptability of 1,3,4-oxadiazole has led to the wide-ranging use of this compound in organic synthesis and have attracted the researcher to work on this type of scaffold and study their biological application.

On the other hand, derivatives of cinnamic acid are important intermediate in organic synthesis and also posses wide range of activity such as antimicrobial [12], anti allergic [13], antitubulin [14], anticancer [15], antioxidant [16,17]. Using the cinnamic hydrazide and triethyl orthoester, microwave assisted synthesis of styryl-1,3,4-oxadiazoles have been reported by Kudelko and Zieliski [18-24].

Therefore, considering that the cinnamic acid derivatives and 1,3,4-oxadiazole are important building blocks for the development of new series of heterocycles for 
pharmacological interest, we have embarked upon development of various new 1,3,4oxadiazole derivatives which have been described in following sections.

\section{EXPERIMENTAL}

Melting points of the synthesized compounds were taken in open capillaries tubes. The purity of the compounds was checked by thin layer chromatography (TLC). The IR spectra were recorded on a Perkin-Elmer 1800 FTIR spectrometer in $\mathrm{KBr}$ pellets. The ${ }^{1} \mathrm{HNMR}$ spectra were recorded in DMSO- $d_{6}$ solutions on a Bruker Avance Ultra shielded $400 \mathrm{MHz}$ NMR spectrometer using TMS as the internal standard. The mass spectra were recorded on a Shimadzu GCMS-QP 1000 EX. Elemental analysis of the all the synthesized compounds were carried out in Euro EA 3000 Elemental Analyzer and the results are in agreements with the structures assigned. The necessary chemicals were purchased from Spectrochem, Sisco, Loba Chemie, and Sigma Aldrich.

Substituted aromatic aldehyde $(10 \mathrm{~m} \mathrm{~mol})$, malonic acid $(15 \mathrm{~m} \mathrm{~mol})$, piperidine $(4 \mathrm{~m}$ mol) and pyridine as solvent were taken in $100 \mathrm{ml}$ two necked round bottom flask and refluxed for 6 hours. After the completion the reaction, the reaction mixture was allowed to cool ambient temperature and it was poured on to the mixture of 1:1 $\mathrm{HCl}$ : cold water. The separated product was filtered and washes with diluted $\mathrm{HCl}$ and crystallized from methanol. The product was enough pure and taken for next step without further purification. Yield $75 \%$ to $88 \%$.

Cinnamic acid $(0.1 \mathrm{~mol})$ was taken in dried $250 \mathrm{ml} \mathrm{RBF}$ and cooled it to $0-5{ }^{\circ} \mathrm{C}$ then thionyle chloride $(12 \mathrm{ml})$ was added drop wise with the time period of 40 minute and stirred for 1 hour. Excess thionyle chloride was distilled off under reduced pressure and then added hydrazine hydrate $(0.1 \mathrm{~mol})$. After the addition of hydrazine hydrate, reaction mixture was stirred at ambient temperature for 2 hour. The progress and the completion of the reaction were checked by thin layer chromatography. After completion of reaction, reaction mass was poured on to the crushed ice, filtered separated product and washed with cold water. Product was crystallized from glacial $\mathrm{CH}_{3} \mathrm{COOH}$. M.P. $116-118{ }^{\circ} \mathrm{C}$, Yield was $89 \%$.

\section{1. General procedure for the synthesis of 2-((E)-substituted styryl)-5-((E)-styryl-1,3, 4-oxadiazole: (5a-5o).}

In dried $100 \mathrm{ml}$ round bottom flask, compound 4a (cinnamohydrazide $10 \mathrm{mmol}$ ), substituted cinnamic acid $(10 \mathrm{mmol})$ and $\mathrm{POCl}_{3}(15 \mathrm{mmol})$ were taken and subjected to irradiated under microwave (600 watts) for 5 to 10 minutes under the inert condition. The reaction mixture was cooled to ambient temperature and then poured on to crush ice, stirred for 30 minutes at room temperature and filtered. The obtained solids were further neutralized with $50 \mathrm{ml} 10 \%$ sodium bicarbonate solution and wash with $50 \mathrm{ml}$ demineralized water. The resulting compound was purified by column chromatography by silica gel 230-400 mesh using ethyl acetate: hexane (4: $6 \mathrm{v} / \mathrm{v})$ as eluent. Yield: 75-89\%. Physical constants of newly synthesized 2-((E)-substituted styryl)-5-((E)-styryl-1,3,4-oxadiazole derivatives 5a-5o are recorded in Table 1. 


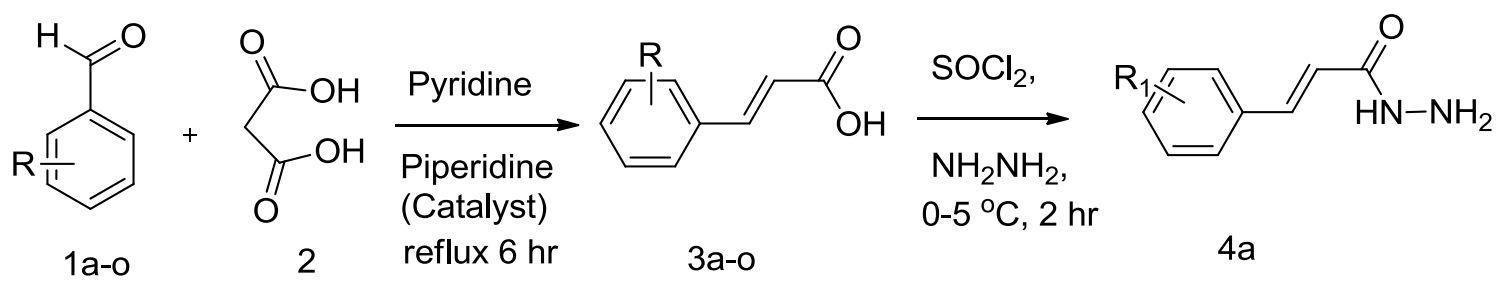

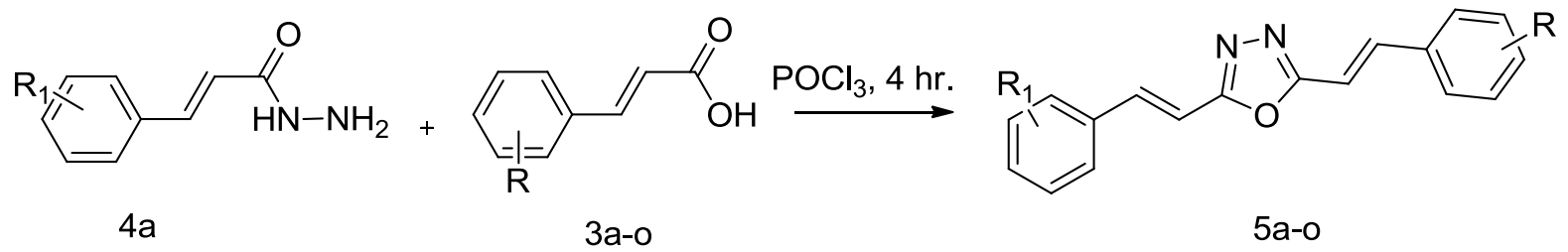

Table 1. Physical data of compounds (5a-5o).

\begin{tabular}{|c|c|c|c|c|c|}
\hline \multirow{2}{*}{ Code } & \multicolumn{2}{|c|}{ Substitution } & \multicolumn{2}{|c|}{$\begin{array}{c}\text { Microwave } \\
\text { Method }\end{array}$} & \multirow{2}{*}{$\underset{{ }^{\circ} \mathrm{C}}{\mathrm{M.P}}$} \\
\hline & $\mathrm{R}$ & $\mathrm{R}_{1}$ & $\begin{array}{l}\text { Time } \\
(\min )\end{array}$ & $\begin{array}{c}\text { Yield } \\
\%\end{array}$ & \\
\hline $5 a$ & $\mathrm{H}$ & $\mathrm{H}$ & 4 & 87 & $162-164$ \\
\hline $5 b$ & $2,4-\mathrm{OCH}_{3}$ & $\mathrm{H}$ & 6 & 79 & $158-160$ \\
\hline $5 c$ & $4-\mathrm{NO}_{2}$ & $\mathrm{H}$ & 7 & 89 & $240-242$ \\
\hline $5 d$ & $4-\mathrm{OC}_{2} \mathrm{H}_{5}$ & $\mathrm{H}$ & 9 & 80 & $152-154$ \\
\hline $5 e$ & $4-\mathrm{OC}_{8} \mathrm{H}_{15}$ & $\mathrm{H}$ & 7 & 78 & $132-130$ \\
\hline $5 f$ & $4-\mathrm{F}$ & $\mathrm{H}$ & 8 & 84 & $166-168$ \\
\hline $5 \mathrm{~g}$ & $4-\mathrm{OH}$ & $\mathrm{H}$ & 10 & 75 & $198-200$ \\
\hline $5 \mathrm{~h}$ & $4-\mathrm{Cl}$ & $\mathrm{H}$ & 6 & 85 & $162-164$ \\
\hline $5 \mathrm{i}$ & $2,5-\mathrm{OCH}_{3}$ & $\mathrm{H}$ & 6 & 78 & 156-158 \\
\hline $5 \mathrm{j}$ & $2-\mathrm{NO}_{2}$ & $\mathrm{H}$ & 6 & 82 & $258-260$ \\
\hline $5 \mathrm{k}$ & $4-\mathrm{OC}_{4} \mathrm{H}_{9}$ & $\mathrm{H}$ & 7 & 83 & $142-144$ \\
\hline 51 & $4-\mathrm{CH}_{3}$ & $\mathrm{H}$ & 12 & 85 & $158-160$ \\
\hline $5 \mathrm{~m}$ & $3,4,5-\mathrm{OCH}_{3}$ & $\mathrm{H}$ & 11 & 76 & $146-148$ \\
\hline $5 n$ & $\begin{array}{c}3-\mathrm{OCH}_{3}, \\
4-\mathrm{OH}\end{array}$ & $\mathrm{H}$ & 9 & 89 & $168-170$ \\
\hline 50 & $2-\mathrm{F}$ & $\mathrm{H}$ & 8 & 87 & $176-178$ \\
\hline
\end{tabular}




\section{2. Analytical data}

\section{2, 5-di((E)-styryl)-1,3,4-oxadiazole (5a)}

MP: $162-164{ }^{\circ} \mathrm{C}$; IR $\left(\mathrm{cm}^{-1}\right): 3045(\mathrm{Ar}-\mathrm{H}$ stretch $), 1680(\mathrm{C}=\mathrm{N}$ stretch of 1,3,4-oxadiazole ring), $2145(\mathrm{C}=\mathrm{C}$ stretching), 1250 ( $\mathrm{C}-\mathrm{O}-\mathrm{C}$ stretch of 1,3,4-oxadiazole ring); $\mathrm{MS}: \mathrm{m} / \mathrm{z}=274$; ${ }^{1} \mathrm{H}$ NMR $\left(\mathrm{CDCl}_{3}\right) \delta$ ppm: $7.05(2 \mathrm{H}, \mathrm{d}, J=15.5 \mathrm{~Hz}), 7.12(2 \mathrm{H}, \mathrm{d}, J=15.5 \mathrm{~Hz}), 7.41-7.52$ $(10 \mathrm{H}, \mathrm{m})$; Elemental Analysis: Calculated for $\mathrm{C}_{18} \mathrm{H}_{14} \mathrm{~N}_{2} \mathrm{O} ; \mathrm{C}, 78.81 ; \mathrm{H}, 5.14 ; \mathrm{N}, 10.21$; O, 5.83 Found: C, 76.36; H, 6.20; N, 9.17; O, 7.48.

\section{2-((E)-2, 4-dimethoxystyryl)-5-((E)-styryl)-1,3,4-oxadiazole (5b)}

MP: $158-160{ }^{\circ} \mathrm{C}$; IR $\left(\mathrm{cm}^{-1}\right)$ : $3052(\mathrm{Ar}-\mathrm{H}$ stretch), $2965(\mathrm{C}-\mathrm{H}$ stretch) $1678(\mathrm{C}=\mathrm{N}$ stretch of 1,3,4-oxadiazole ring), $2148(\mathrm{C}=\mathrm{C}$ stretching), $1254(\mathrm{C}-\mathrm{O}-\mathrm{C}$ stretch of 1,3,4-oxadiazole ring), $1075\left(\mathrm{C}-\mathrm{O}\right.$ stretch); $\mathrm{MS}: \mathrm{m} / \mathrm{z}=334 ;{ }^{1} \mathrm{H}$ NMR $\left(\mathrm{CDCl}_{3}\right) \delta \mathrm{ppm}: 3.75(6 \mathrm{H}, \mathrm{s}), 6.98(1 \mathrm{H}$, $\mathrm{d}, J=15.4 \mathrm{~Hz}), 6.70(1 \mathrm{H}, \mathrm{d}, J=15.4 \mathrm{~Hz}), 6.46(1 \mathrm{H}, \mathrm{d}, J=15.8 \mathrm{~Hz}), 7.17(1 \mathrm{H}, \mathrm{d}, J=15.8$ $\mathrm{Hz})$, 7.52-7.68 (9H, m); Elemental Analysis: Calculated for $\mathrm{C}_{20} \mathrm{H}_{18} \mathrm{~N}_{2} \mathrm{O}_{3} ; \mathrm{C}, 71.84 ; \mathrm{H}, 5.43$; N, 8.38; O, 14.35 Found: C, 70.64; H, 5.33; N, 7.95; O, 16.35.

\section{2-((E)-4-nitrostyryl)-5-((E)-styryl)-1,3,4-oxadiazole) (5c)}

MP: $240-242{ }^{\circ} \mathrm{C}$; IR $\left(\mathrm{cm}^{-1}\right)$ : 3052 (Ar-H stretch), $1685(\mathrm{C}=\mathrm{N}$ stretch of 1,3,4-oxadiazole ring), $2156(\mathrm{C}=\mathrm{C}$ stretching), $1255(\mathrm{C}-\mathrm{O}-\mathrm{C}$ stretch of 1,3,4-oxadiazole ring), 1366 (Assymetric $\mathrm{N}-\mathrm{O}$ stretch of $\left.\mathrm{C}-\mathrm{NO}_{2}\right) ; \mathrm{MS}: \mathrm{m} / \mathrm{z}=319 ;{ }^{1} \mathrm{H} \mathrm{NMR}\left(\mathrm{CDCl}_{3}\right) \delta \mathrm{ppm}: 6.98(1 \mathrm{H}, \mathrm{d}$, $J=15.3 \mathrm{~Hz}), 6.72(1 \mathrm{H}, \mathrm{d}, J=15.3 \mathrm{~Hz}), 6.92(1 \mathrm{H}, \mathrm{d}, J=15.6 \mathrm{~Hz}), 7.13(1 \mathrm{H}, \mathrm{d}, J=15.6 \mathrm{~Hz})$, 7.39-7.47 (5H, m), 7.59-7.67 (4H, d); Elemental Analysis: Calculated for $\mathrm{C}_{18} \mathrm{H}_{13} \mathrm{~N}_{3} \mathrm{O}_{3}$; C, 67.71; H, 4.10; N, 13.16; O, 15.03 Found: - C, 67.80; H, 4.25; N, 12.96; O, 15.12.

\section{2-((E)-4-ethoxystyryl)-5-((E)-styryl)-1,3,4-oxadiazole (5d)}

MP: $152-154{ }^{\circ} \mathrm{C}$; IR $\left(\mathrm{cm}^{-1}\right)$ : 3042 (Ar-H stretch), 2981 (C-H stretch), 1679 (C=N stretch of 1,3,4-oxadiazole ring), $2145(\mathrm{C}=\mathrm{C}$ stretching), $1260(\mathrm{C}-\mathrm{O}-\mathrm{C}$ stretch of 1,3,4-oxadiazole ring), 1082 (C-O stretch); MS: $\mathrm{m} / \mathrm{z}=318 ;{ }^{1} \mathrm{H} \mathrm{NMR}\left(\mathrm{CDCl}_{3}\right) \delta \mathrm{ppm}: 1.30(3 \mathrm{H}, \mathrm{t}), 3.89(2 \mathrm{H}$, q), $6.99(1 \mathrm{H}, \mathrm{d}, J=15.1 \mathrm{~Hz}), 6.71(1 \mathrm{H}, \mathrm{d}, J=15.1 \mathrm{~Hz}), 6.65(1 \mathrm{H}, \mathrm{d}, J=15.4 \mathrm{~Hz}), 7.01(1 \mathrm{H}, \mathrm{d}$, $J=15.4 \mathrm{~Hz}), 7.95-8.12(9 \mathrm{H}, \mathrm{m})$; Elemental Analysis: Calculated for $\mathrm{C}_{20} \mathrm{H}_{18} \mathrm{~N}_{2} \mathrm{O}_{2} ; \mathrm{C}, 75.45$; H, 5.70; N, 8.80; O, 10.05 Found: C, 75.80; H, 4.96; N, 9.00; O, 11.05.

\section{2-((E)-4-(n-octyloxy)styryl)-5-((E)-styryl)-1,3,4-oxadiazole (5e)}

MP: $132-130{ }^{\circ} \mathrm{C}$; IR $\left(\mathrm{cm}^{-1}\right): 3062(\mathrm{Ar}-\mathrm{H}$ stretch), $2975(\mathrm{C}-\mathrm{H}$ stretch), $1675(\mathrm{C}=\mathrm{N}$ stretch of 1,3,4-oxadiazole ring), $2150(\mathrm{C}=\mathrm{C}$ stretching $), 1250(\mathrm{C}-\mathrm{O}-\mathrm{C}$ stretch of 1,3,4-oxadiazole ring), $1080\left(\mathrm{C}-\mathrm{O}\right.$ stretch); MS: $\mathrm{m} / \mathrm{z}=400 ;{ }^{1} \mathrm{H}$ NMR $\left(\mathrm{CDCl}_{3}\right) \delta \mathrm{ppm}: 1.12(3 \mathrm{H}, \mathrm{t}), 2.54(2 \mathrm{H}$, t), 2.60-2.82 (7H, m), $4.22(2 \mathrm{H}, \mathrm{d}), 7.21(1 \mathrm{H}, \mathrm{d}, J=15.2 \mathrm{~Hz}), 6.98(1 \mathrm{H}, \mathrm{d}, J=15.2 \mathrm{~Hz}), 7.01$ $(1 \mathrm{H}, \mathrm{d}, J=15.9 \mathrm{~Hz}), 7.25(1 \mathrm{H}, \mathrm{d}, J=15.9 \mathrm{~Hz}), 7.35-7.48(9 \mathrm{H}, \mathrm{m})$; Elemental Analysis: Calculated for $\mathrm{C}_{26} \mathrm{H}_{28} \mathrm{~N}_{2} \mathrm{O}_{2} ; \mathrm{C}, 77.97 ; \mathrm{H}, 7.05 ; \mathrm{N}, 6.99 ; \mathrm{O}, 7.99$ Found: C, 76.07; H, 6.12; N, 7.09; O, 8.02.

\section{2-((E)-4-fluorostyryl)-5-((E)-styryl)-1,3,4-oxadiazole (5f)}

MP: $166-168{ }^{\circ} \mathrm{C}$; IR $\left(\mathrm{cm}^{-1}\right): 3060(\mathrm{Ar}-\mathrm{H}$ stretch $), 1684(\mathrm{C}=\mathrm{N}$ stretch of 1,3,4-oxadiazole ring), $2164(\mathrm{C}=\mathrm{C}$ stretching), $1258(\mathrm{C}-\mathrm{O}-\mathrm{C}$ stretch of 1,3,4-oxadiazole ring $), 1088(\mathrm{C}-\mathrm{O}$ stretch) 740,1352 (C-Fstretch); MS: $\mathrm{m} / \mathrm{z}=292 ;{ }^{1} \mathrm{H} \mathrm{NMR}\left(\mathrm{CDCl}_{3}\right) \delta \mathrm{ppm}: 7.0(1 \mathrm{H}, \mathrm{d}, J=15.5$ 
$\mathrm{Hz}), 6.71(1 \mathrm{H}, \mathrm{d}, J=15.5 \mathrm{~Hz}), 6.66(1 \mathrm{H}, \mathrm{d}, J=15.1 \mathrm{~Hz}), 7.14(1 \mathrm{H}, \mathrm{d}, J=15.1 \mathrm{~Hz}), 7.54-7.68$ $(9 \mathrm{H}, \mathrm{m})$; Elemental Analysis: Calculated for $\mathrm{C}_{18} \mathrm{H}_{13} \mathrm{FN}_{2} \mathrm{O} ; \mathrm{C}, 73.96 ; \mathrm{H}, 4.48 ; \mathrm{F}, 6.50 ; \mathrm{N}, 9.58$; O, 5.47 Found: C, 73.62; H, 5.09; F, 6.25; N, 9.40; O, 6.10.

\section{4-((E)-2-(5-((E)-styryl)-1,3,4-oxadiazol-2-yl)vinyl)phenol (5g)}

MP: $198-200{ }^{\circ} \mathrm{C}$; IR $\left(\mathrm{cm}^{-1}\right)$ : $3454(\mathrm{O}-\mathrm{H}$ stretch) $3058(\mathrm{Ar}-\mathrm{H}$ stretch), $1684(\mathrm{C}=\mathrm{N}$ stretch of 1,3,4-oxadiazole ring), $2145(\mathrm{C}=\mathrm{C}$ stretching), $1248(\mathrm{C}-\mathrm{O}-\mathrm{C}$ stretch of 1,3,4-oxadiazole ring); $\mathrm{MS}: \mathrm{m} / \mathrm{z}=290 ;{ }^{1} \mathrm{H} \mathrm{NMR}\left(\mathrm{CDCl}_{3}\right) \delta \mathrm{ppm}: 6.02(1 \mathrm{H}, \mathrm{d}, J=15.3 \mathrm{~Hz}), 7.04(1 \mathrm{H}, \mathrm{d}, J=$ $15.3 \mathrm{~Hz}), 6.70(1 \mathrm{H}, \mathrm{d}, J=15.8 \mathrm{~Hz}), 6.65(1 \mathrm{H}, \mathrm{d}, J=15.7 \mathrm{~Hz}), 7.06(1 \mathrm{H}, \mathrm{d}, J=15.7 \mathrm{~Hz})$, 7.64-7.92 (9H, m); Elemental Analysis: Calculated for $\mathrm{C}_{18} \mathrm{H}_{14} \mathrm{~N}_{2} \mathrm{O}_{2} ; \mathrm{C}, 74.47 ; \mathrm{H}, 4.86$; N, 9.65; O, 11.02 Found: C, 74.11; H, 5.96; N, 9.86; O, 12.08.

\section{2-((E)-4-chlorostyryl)-5-((E)-styryl)-1,3,4-oxadiazole (5h)}

MP: $162-164{ }^{\circ} \mathrm{C}$; IR $\left(\mathrm{cm}^{-1}\right)$ : $3056(\mathrm{Ar}-\mathrm{H}$ stretch), $1685(\mathrm{C}=\mathrm{N}$ stretch of 1,3,4-oxadiazole ring), $2148(\mathrm{C}=\mathrm{C}$ stretching), $1256(\mathrm{C}-\mathrm{O}-\mathrm{C}$ stretch of 1,3,4-oxadiazole ring), 552,890 (C-Cl stretch); MS: $\mathrm{m} / \mathrm{z}=308 ;{ }^{1} \mathrm{H}$ NMR $\left(\mathrm{CDCl}_{3}\right) \delta \mathrm{ppm}: 6.07(1 \mathrm{H}, \mathrm{d}, J=15.5 \mathrm{~Hz}), 7.12(1 \mathrm{H}, \mathrm{d}, J$ $=15.5 \mathrm{~Hz}), 6.75(1 \mathrm{H}, \mathrm{d}, J=15.9 \mathrm{~Hz}), 6.70(1 \mathrm{H}, \mathrm{d}, J=15.8 \mathrm{~Hz}), 7.06(1 \mathrm{H}, \mathrm{d}, J=15.7 \mathrm{~Hz})$, 7.74-7.95 (8H, m); Elemental Analysis: Calculated for $\mathrm{C}_{18} \mathrm{H}_{13} \mathrm{ClN}_{2} \mathrm{O} ; \mathrm{C}, 70.02 ; \mathrm{H}, 4.24 ; \mathrm{Cl}$, 11.48; N, 9.07; O, 5.18 Found: C, 71.00; H, 4.24; Cl, 11.48; N, 9.07; O, 5.18.

\section{2-((E)-2,5-dimethoxystyryl)-5-((E)-styryl)-1,3,4-oxadiazole (5i)}

MP: $156-158{ }^{\circ} \mathrm{C}$; IR $\left(\mathrm{cm}^{-1}\right)$ : $3052($ Ar-H stretch), $2860(\mathrm{C}-\mathrm{H}$ stretch), $1682(\mathrm{C}=\mathrm{N}$ stretch of 1,3,4-oxadiazole ring), $2162(\mathrm{C}=\mathrm{C}$ stretching), $1260(\mathrm{C}-\mathrm{O}-\mathrm{C}$ stretch of 1,3,4-oxadiazole ring), $1086\left(\mathrm{C}-\mathrm{O}\right.$ stretch); MS: $\mathrm{m} / \mathrm{z}=334 ;{ }^{1} \mathrm{H}$ NMR $\left(\mathrm{CDCl}_{3}\right) \delta \mathrm{ppm}: 3.76(6 \mathrm{H}, \mathrm{s}), 7.02(1 \mathrm{H}, \mathrm{d}$, $J=15.4 \mathrm{~Hz}), 6.71(1 \mathrm{H}, \mathrm{d}, J=15.4 \mathrm{~Hz}), 6.51(1 \mathrm{H}, \mathrm{d}, J=15.7 \mathrm{~Hz}), 7.19(1 \mathrm{H}, \mathrm{d}, J=15.7 \mathrm{~Hz})$, $7.25(2 \mathrm{H}, \mathrm{m}), 7.56-7.89(6 \mathrm{H}, \mathrm{m})$; Elemental Analysis: Calculated for $\mathrm{C}_{20} \mathrm{H}_{18} \mathrm{~N}_{2} \mathrm{O}_{3} ; \mathrm{C}, 71.84$; H, 5.43; N, 8.38; O, 14.35 Found: C, 71.65; H, 4.83; N, 8.58; O, 15.20

\section{2-((E)-2-nitrostyryl)-5-((E)-styryl)-1,3,4-oxadiazole (5j)}

MP: $258-260{ }^{\circ} \mathrm{C}$; IR $\left(\mathrm{cm}^{-1}\right)$ : $3110(\mathrm{Ar}-\mathrm{H}$ stretch $), 1678(\mathrm{C}=\mathrm{N}$ stretch of 1,3,4-oxadiazole ring),2175 $(\mathrm{C}=\mathrm{C} \quad$ stretching $), 1282(\mathrm{C}-\mathrm{O}-\mathrm{C}$ stretch of 1,3,4-oxadiazole ring), 1421 (Assymetric $\mathrm{N}-\mathrm{O}$ stretch of $\left.\mathrm{C}-\mathrm{NO}_{2}\right) ; \mathrm{MS}: \mathrm{m} / \mathrm{z}=319 ;{ }^{1} \mathrm{H} \mathrm{NMR}\left(\mathrm{CDCl}_{3}\right) \delta \mathrm{ppm}: 7.00(1 \mathrm{H}, \mathrm{d}$, $J=15.4 \mathrm{~Hz}), 6.07(1 \mathrm{H}, \mathrm{d}, J=15.4 \mathrm{~Hz}), 6.62(1 \mathrm{H}, \mathrm{d}, J=15.9 \mathrm{~Hz}), 7.54(1 \mathrm{H}, \mathrm{d}, J=15.9 \mathrm{~Hz})$, 7.40-7.51 (5H, m), 7.59-7.62 $(4 \mathrm{H}, \mathrm{d})$; Elemental Analysis: Calculated for $\mathrm{C}_{18} \mathrm{H}_{13} \mathrm{~N}_{3} \mathrm{O}_{3} ; \mathrm{C}$, 67.71; H, 4.10; N, 13.16; O, 15.03 Found: C, 67.92; H, 4.86; N, 12.56; O, 15.88.

\section{2-((E)-4-butoxystyryl)-5-((E)-styryl)-1,3,4-oxadiazole (5k)}

MP: $142-144{ }^{\circ} \mathrm{C}$; IR $\left(\mathrm{cm}^{-1}\right): 3083$ (Ar-H stretch), 2946 (C-H stretch), $1688(\mathrm{C}=\mathrm{N}$ stretch of 1,3,4-oxadiazole ring), $2182(\mathrm{C}=\mathrm{C}$ stretching), $1274(\mathrm{C}-\mathrm{O}-\mathrm{C}$ stretch of 1,3,4-oxadiazole ring), 1132 (C-O stretch); MS: $\mathrm{m} / \mathrm{z}=346 ;{ }^{1} \mathrm{H}$ NMR $\left(\mathrm{CDCl}_{3}\right) \delta \mathrm{ppm}: 1.12(3 \mathrm{H}, \mathrm{t}), 1.48(2 \mathrm{H}$, q), $1.78(2 \mathrm{H}, \mathrm{q}), 4.51(2 \mathrm{H}, \mathrm{t}), 7.00(1 \mathrm{H}, \mathrm{d}, J=15.2 \mathrm{~Hz}), 6.71(1 \mathrm{H}, \mathrm{d}, J=15.2 \mathrm{~Hz}), 6.64(1 \mathrm{H}$, d, $J=15.9 \mathrm{~Hz}), 6.99(1 \mathrm{H}, \mathrm{d}, J=15.9 \mathrm{~Hz}), 6.97-7.72(9 \mathrm{H}, \mathrm{m})$; Elemental Analysis: Calculated for $\mathrm{C}_{22} \mathrm{H}_{22} \mathrm{~N}_{2} \mathrm{O}_{2} ; \mathrm{C}, 76.28 ; \mathrm{H}, 6.40 ; \mathrm{N}, 8.09 ; \mathrm{O}, 9.24$ Found: $\mathrm{C}, 76.37 ; \mathrm{H}, 6.90 ; \mathrm{N}, 8.51 ; \mathrm{O}$, 10.16 . 


\section{2-((E)-4-methylstyryl)-5-((E)-styryl)-1,3,4-oxadiazole (5l)}

MP: $158-160{ }^{\circ} \mathrm{C}$; IR $\left(\mathrm{cm}^{-1}\right): 3060(\mathrm{Ar}-\mathrm{H}$ stretch), $2889(\mathrm{C}-\mathrm{H}$ stretch) $1658(\mathrm{C}=\mathrm{N}$ stretch of 1,3,4-oxadiazole ring), $2158(\mathrm{C}=\mathrm{C}$ stretching), $1260(\mathrm{C}-\mathrm{O}-\mathrm{C}$ stretch of 1,3,4-oxadiazole ring); $\mathrm{MS}: \mathrm{m} / \mathrm{z}=288 ;{ }^{1} \mathrm{H}$ NMR $\left(\mathrm{CDCl}_{3}\right) \delta \mathrm{ppm}: 2.10(3 \mathrm{H}, \mathrm{s}), 7.01(1 \mathrm{H}, \mathrm{d}, J=15.1 \mathrm{~Hz}), 6.72$ $(1 \mathrm{H}, \mathrm{d}, J=15.1 \mathrm{~Hz}), 6.67(1 \mathrm{H}, \mathrm{d}, J=15.6 \mathrm{~Hz}), 7.02(1 \mathrm{H}, \mathrm{d}, J=15.6 \mathrm{~Hz}), 7.48-7.56(9 \mathrm{H}, \mathrm{m})$; Elemental Analysis: Calculated for $\mathrm{C}_{19} \mathrm{H}_{16} \mathrm{~N}_{2} \mathrm{O} ; \mathrm{C}, 79.14 ; \mathrm{H}, 5.59 ; \mathrm{N}, 9.72 ; \mathrm{O}, 5.55$ Found: $\mathrm{C}$, 79.82; H, 4.49; N, 8.71; O, 7.12.

\section{2-((E)-styryl)-5-((E)-3,4,5-trimethoxystyryl)-1,3,4-oxadiazole (5m)}

MP: $146-148{ }^{\circ} \mathrm{C}$; IR $\left(\mathrm{cm}^{-1}\right)$ : 3124 (Ar-H stretch), $2966(\mathrm{C}-\mathrm{H}$ stretch) $1645(\mathrm{C}=\mathrm{N}$ stretch of 1,3,4-oxadiazole ring), $2171(\mathrm{C}=\mathrm{C}$ stretching $), 1264(\mathrm{C}-\mathrm{O}-\mathrm{C}$ stretch of 1,3,4-oxadiazole ring), $1085\left(\mathrm{C}-\mathrm{O}\right.$ stretch); MS: $\mathrm{m} / \mathrm{z}=364 ;{ }^{1} \mathrm{H}$ NMR $\left(\mathrm{CDCl}_{3}\right) \delta \mathrm{ppm}: 4.12(9 \mathrm{H}, \mathrm{s}), 7.04(1 \mathrm{H}$, $\mathrm{d}, J=15.3 \mathrm{~Hz}), 6.72(1 \mathrm{H}, \mathrm{d}, J=15.3 \mathrm{~Hz}), 6.71(1 \mathrm{H}, \mathrm{d}, J=15.8 \mathrm{~Hz}), 7.06(1 \mathrm{H}, \mathrm{d}, J=15.8$ $\mathrm{Hz})$, 7.64-7.78 (7H, m); Elemental Analysis: Calculated for $\mathrm{C}_{21} \mathrm{H}_{20} \mathrm{~N}_{2} \mathrm{O}_{4} ; \mathrm{C}, 69.22 ; \mathrm{H}, 5.53$; N, 7.69; O, 17.56 Found: C, 69.32; H, 5.64; N, 6.29; O, 18.55

\section{2-methoxy-4-((E)-2-(5-((E)-styryl)-1,3,4-oxadiazol-2-yl)vinyl)phenol (5n)}

MP: $168-170{ }^{\circ} \mathrm{C}$; IR $\left(\mathrm{cm}^{-1}\right)$ : 3458 (O-H stretch), 3072 (Ar-H stretch), 2935 (C-H stretch) $1670(\mathrm{C}=\mathrm{N}$ stretch of 1,3,4-oxadiazole ring), $2164(\mathrm{C}=\mathrm{C}$ stretching $), 1310(\mathrm{C}-\mathrm{O}-\mathrm{C}$ stretch of 1,3,4-oxadiazole ring), 1108 (C-O stretch); $\mathrm{MS}: \mathrm{m} / \mathrm{z}=320 ;{ }^{1} \mathrm{H} \mathrm{NMR}\left(\mathrm{CDCl}_{3}\right) \delta \mathrm{ppm}: 3.71$ $(3 \mathrm{H}, \mathrm{s}), 6.998(1 \mathrm{H}, \mathrm{d}, J=15.2 \mathrm{~Hz}), 6.69(1 \mathrm{H}, \mathrm{d}, J=15.2 \mathrm{~Hz}), 6.65(1 \mathrm{H}, \mathrm{d}, J=15.9 \mathrm{~Hz}), 7.00$ $(1 \mathrm{H}, \mathrm{d}, J=15.9 \mathrm{~Hz}), 7.43-7.78(8 \mathrm{H}, \mathrm{m})$; Elemental Analysis: Calculated for $\mathrm{C}_{19} \mathrm{H}_{16} \mathrm{~N}_{2} \mathrm{O}_{3} ; \mathrm{C}$, 71.24; H, 5.03; N, 8.74; O, 14.98 Found: C, 72.24; H, 6.05; N, 7.71; O, 13.99.

\section{2-((E)-2-fluorostyryl)-5-((E)-styryl)-1,3,4-oxadiazole (5o)}

MP: $176-178{ }^{\circ} \mathrm{C}$; IR $\left(\mathrm{cm}^{-1}\right)$ : $3076(\mathrm{Ar}-\mathrm{H}$ stretch), $1675(\mathrm{C}=\mathrm{N}$ stretch of 1,3,4-oxadiazole ring), $2168(\mathrm{C}=\mathrm{C}$ stretching), $1275(\mathrm{C}-\mathrm{O}-\mathrm{C}$ stretch of 1,3,4-oxadiazole ring), 752,1386 (CFstretch); MS: $\mathrm{m} / \mathrm{z}=292 ;{ }^{1} \mathrm{H}$ NMR $\left(\mathrm{CDCl}_{3}\right) \delta \mathrm{ppm}: 6.97(1 \mathrm{H}, \mathrm{d}, J=15.4 \mathrm{~Hz}), 6.71(1 \mathrm{H}, \mathrm{d}, J$ $=15.4 \mathrm{~Hz}), 6.57(1 \mathrm{H}, \mathrm{d}, J=15.8 \mathrm{~Hz}), 7.20(1 \mathrm{H}, \mathrm{d}, J=15.8 \mathrm{~Hz}), 7.40-7.68(9 \mathrm{H}, \mathrm{m})$; Elemental Analysis: Calculated for $\mathrm{C}_{18} \mathrm{H}_{13} \mathrm{FN}_{2} \mathrm{O} ; \mathrm{C}, 73.96 ; \mathrm{H}, 4.48 ; \mathrm{F}, 6.50 ; \mathrm{N}, 9.58 ; \mathrm{O}, 5.47$ Found: C, 74.76; H, 5.98; F, 5.80; N, 9.71; O, 6.4

\section{ANTIMICROBIAL ACTIVITY}

The all synthesized compounds (5a-o) were screened for in vitro antimicrobial activity against the gram negative bacteria (Escherichia coli) and the gram positive bacteria (Staphylococcus aurous and Bacillus subtitles), expressed plate agar diffusion method.

Comparisons of inhibition zone in bacterial culture by the synthesized compounds were studied by using ofloxacin as a standard drug, at $100 \mu \mathrm{g} / \mathrm{ml}$ concentration. Dimethyl sulphoxide (DMSO) was used to prepare stock and standard solution of compounds (5a-o) and ofloxacin. Bacterial cultures of Bacillus subtilis, Staphylococcus aureus, and Escherichia coli were prepared in the nutrient agar medium. Discs of whatman filter paper $(9 \mathrm{~cm}$ diameter) were moistened with $100 \mu \mathrm{g} / \mathrm{ml}$ solution of the compounds and discs were carefully placed on bacterial culture plates that had been previously inoculated separately with the microorganism. The bacterial cultures were incubated at $37{ }^{\circ} \mathrm{C}$ for 24 hours. The radius of 
zone of inhibition (in $\mathrm{mm}$ ) was observed around the cup after respective incubation was secure and measured in triplicate sets by using a scale.

Table 2. Antimicrobial activity of 2,5-distyryl-1,3,4-oxadiazole derivatives (5a-5o).

\begin{tabular}{|c|c|c|c|}
\hline \multirow{3}{*}{$\begin{array}{l}\text { Compound } \\
\text { code }\end{array}$} & \multicolumn{3}{|c|}{ Zone of inhibition (mm) } \\
\hline & \multicolumn{2}{|c|}{ Gram Positive } & \multirow{2}{*}{$\begin{array}{c}\text { Gram } \\
\text { Negative } \\
\text { Escherichia } \\
\text { coli }\end{array}$} \\
\hline & $\begin{array}{c}\text { Staphylococcus } \\
\text { aureus }\end{array}$ & $\begin{array}{l}\text { Pseudomonas } \\
\text { aeruginosa }\end{array}$ & \\
\hline $5 a$ & 09 & 08 & 09 \\
\hline $5 b$ & 10 & 13 & 11 \\
\hline $5 c$ & 14 & 11 & 10 \\
\hline $5 d$ & 11 & 14 & 12 \\
\hline $5 e$ & 13 & 16 & 13 \\
\hline $5 f$ & 15 & 12 & 15 \\
\hline $5 \mathrm{~g}$ & 18 & 10 & 14 \\
\hline $5 \mathrm{~h}$ & 20 & 12 & 16 \\
\hline $5 \mathrm{i}$ & 10 & 14 & 12 \\
\hline $5 \mathrm{j}$ & 13 & 11 & 11 \\
\hline $5 \mathrm{k}$ & 13 & 15 & 12 \\
\hline 51 & 12 & 12 & 09 \\
\hline $5 \mathrm{~m}$ & 12 & 14 & 10 \\
\hline $5 n$ & 18 & 11 & 14 \\
\hline 50 & 15 & 12 & 16 \\
\hline $\begin{array}{l}\text { Ofloxacin } \\
\text { Standard }\end{array}$ & 41 & 38 & 37 \\
\hline
\end{tabular}

\section{CONCLUSION}

In present report, we have developed an efficient, simple, rapid and eco-friendly microwave- indused method for preparation of asymmetric 2,5-disyryl-1,3,4-oxadiazole derivatives which give the higher yield and also redused the reaction time. From the result of biological data, compound 5g, 5h, 5n, showed excellent activity against Staphylococcus aureus, while compound 5e and 5k showed maximum bacterial activity against Pseudomonas 
aeruginosa and compound 5o, 5h maximum activity against Escherichia coli. Antibacterial activity was compared with ofloxacin as a standard drug. All the compounds were less potent than the standard drugs ofloxacin. The compound having the halogen group shows greater antimicrobial activity.

\section{ACKNOWLEDGEMENT}

We are thanks to the Department of Chemistry (DST-FIST Funded \& UGC-SAP Sponsored), Saurashtra University, Rajkot, for research work. Specially indebted to "National Facility for Drug Discovery through New Chemical Entities (NCE's), Development \& Instrumentation Support to Small Manufacturing Pharma Enterprises", a programme under Drug \& Pharma Research Support (DPRS) jointly funded by Department of Science \& Technology New Delhi.

\section{References}

[1] S. Chao, X. Li, S. Wang, Huaxue Yanjiu Yu Yingyong. 22(8) (2010) 1066-1071.

[2] B. Chandrakantha, Prakash Shetty, Vijesh Nambiyar, Nishitha Isloor, Arun M. Isloor, European Journal of Medicinal Chemistry 45 (2010) 1206-1210.

[3] Rakesh Kumar, M. Shahar Yar, A. K. Rail, Saurabh Chaturvedi, Der Pharmacia Lettre 5 (1) (2013) 366-370.

[4] N. Jain, D. P. Pathak, P. Mishra, S. Jain, J. Iranian Chem. Soc. 6 (2009) 77.

[5] L. Srikanth, U. Naik, R. Jadhav, N. Raghunandan, J. Rao, K. Manohar., Pharma Chemica 2(4) (2010) 231-243.

[6] M. Bhat, M. Al-Omar, N. Siddiqui, Pharma Chemica 2(2) (2010) 1-10.

[7] K. Sushil, V. Gupta, V. Kashaw, P. Mishra, J. Stables, N. Jain., Med. Chem. Research. 38(2) (2009) 157-159.

[8] L. Jin, J. Chen, B. Song, Z. Chen, S. Yang, Q. Li, D. Hu, R. Xu, Bioorg. Med. Chem. Lett. 16 (2006) 5036.

[9] Wang Bao-Lei, Li Zheng-Ming, Li Yong-Hong, Wang Su-Hua, Gaodeng Xuexia o Ниахие Хиеbаo 29(1) (2008) 90-94.

[10] C. R. W. Guimaraes, D. L. Boger, W. L. Jorgensen, J. Am. Chem. Soc. 127 (2005) 17377-17384.

[11] B. Narayana, B. V. Ashalatha,a K. K. Vijaya Raj, J. Fernandes and B. K. Sarojini, Bioorganic \& Medicinal Chemistry 13 (2005) 4638-4644.

[12] Prateek Sharma, J. Chem. Pharm. Res. 3(2) (2005) 403-423

[13] Rolando F. Pellon, Taimirys Mamposo, Erick Godlez, Osmar Calderon, Synthetic Communications 30(20) (2000) 3769-3774.

[14] Yin Luo, Ke-Ming Qiu, Xiang Lu, Kai Liu, Jie Fu, Hai-Liang Zhu, Bioorganic \& Medicinal Chemistry 19(16) (2011) 4730-4738.

[15] Lei Liu, W. Robert Hudgins, Sonsoles Shack, Mu Quan Yin, Dvorit Samid, International Journal of Cancer 62(3) (1995) 345-350. 
[16] Fausta Natella, Mirella Nardini, Maurizio Di Felice, Cristina Scaccini, J. Agric. Food Chem. 47(4) (1999) 1453-1459.

[17] Mario Foti, Mario Piattelli, Maria Tiziana Baratta, Giuseppe Ruberto, J. Agric. Food Chem. 44(2) (1996) 497-501.

[18] Kudelko A., Zieliński W., Tetrahedron Lett. 53 (2012) 76-77.

[19] Rahul R. Tripathi, Ratnamala P. Sonawane, International Letters of Chemistry, Physics and Astronomy 10(2) (2013) 119-125.

[20] Mallikarjun S. Yadawe, Shrishila N. Unki, Sangamesh A. Patil, International Letters of Chemistry, Physics and Astronomy 12 (2013) 94-104.

[21] G. Thirunarayanan, International Letters of Chemistry, Physics and Astronomy 5 (2014) 89-98.

[22] V. J. Faldu, P. K. Talpara, N. H. Bhuva, P. R. Vachharajani, V. H. Shah, International Letters of Chemistry, Physics and Astronomy 6 (2014) 26-32.

[23] Piyush B. Vekariya, Jalpa R. Pandya, Vaishali Goswami, Hitendra S. Joshi, International Letters of Chemistry, Physics and Astronomy 7 (2014) 45-52.

[24] R. G. Vaghasiya, H. B. Ghodasara, P. R. Vachharajani, V. H. Shah, International Letters of Chemistry, Physics and Astronomy 8 (2014) 30-37. 